\title{
Ligandeneffekte bei der diastereoselektiven Addition von Organocerverbindung an Carbonyl- verbindungen**
}

\section{Carmen Alcaraz und Ulrich Groth*}

Eine der wichtigsten Anwendungen der Lanthanoide in der präparativen Organischen Chemie ist die Addition von Organocerreagentien an Carbonylverbindungen. ${ }^{[1]}$ Aufgrund ihrer geringen Basizität und hohen Nucleophilie führen sie bei der Reaktion mit leicht enolisierbaren Substraten $\mathrm{zu}$ höheren Ausbeuten als die entsprechenden Organolithium- oder Orga-

[*] Prof, Dr. U. Groth, Dr. C. Alcaraz

Fakultät für Chemie der Universität

Universitätsstraße 10, D-78457 Konstanz

Telefax: Int. + 7531/884155

E-mail: Ulrich.Groth $(a)$ uni-konstanz.de

[**] Lanthanoide in der Organischen Synthese, 2. Mitteilung. Diese Arbeit wurde von der EU-Kommission, Directorate General XIl, und vom Fonds der Chemischen Industrie gefördert. C. A dankt der EU-Kommission für ein Individualstipendium (Nr. ERBCHBI-CT94-1465). - 1. Mitteilung: Lit. [4 b] 
nomagnesiumreagentien. Außerdem dürften Organocerverbindungen aus sterischen Gründen geeignet sein, eine hohe Diastereoselektivität bei der Addition an Carbonylverbindungen zu bewirken. ${ }^{[2]}$ Die Art der Liganden bestimmt die Richtung und das Ausmaß der Stereoselektivität bei vielen Carbonyladditionen $;^{[3]}$ bei der Addition von Organocerreagentien an chirale Carbonylverbindungen wurde dies bis jetzt noch nicht systematisch untersucht.

Wir berichten hier über die Synthese neuer Alkoxy- und Amidocerverbindungen der allgemeinen Formeln $\mathrm{ClCe}(\mathrm{OR})_{2}$, $\mathrm{Ce}(\mathrm{OR})_{3}$ und $\mathrm{Ce}\left(\mathrm{NR}_{2}\right)_{3}$. Die $\mathrm{Cer}(\mathrm{III})$ trialkoxide waren durch Ligandenaustausch des bekannten $\mathrm{Ce}(\mathrm{OiPr})_{3}{ }^{[3 c, 4]}$ mit den entsprechenden Alkoholen ${ }^{[5]}$ [Gl. (a)] zugänglich. Die Cer(III)chloriddialkoxide wurden durch Reaktion der jeweiligen Cer(III)trialkoxide mit je einem $\ddot{\text { Aquivalent Acetylchlorid }}{ }^{[6]}$ erhalten [Gl. (b)] und die Amidocerverbindungen durch Reaktion von $\mathrm{CeCl}_{3}$. THF mit drei Äquivalenten der entsprechenden Lithiumamide $^{[7]}$ [Gl. (c)]. Diese neuen Alkoxy- und Amidocerverbindungen wurden ohne weitere Reinigung eingesetzt. Aufgrund ihrer geringen Kristallisationstendenz konnten von ihnen bisher keine Röntgenstrukturanalysen angefertigt werden.

$\mathrm{Ce}(\mathrm{O} / \mathrm{Pr})_{3}+3 \mathrm{ROH}$

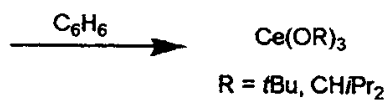

$\mathrm{Ce}(\mathrm{OR})_{3}+\mathrm{CH}_{3} \mathrm{COCl}$

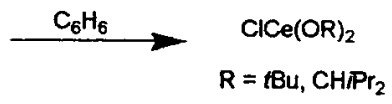

$\mathrm{CeCl}_{3} \cdot \mathrm{THF}+3 \mathrm{LiNR}_{2}$

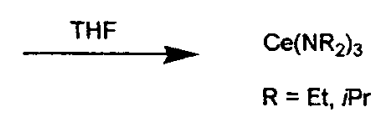

Für die Addition wurde als Substrat das Cyclopentanon rac-1 verwendet, da bekannt ist, daß die Diastereoselektivitäten bei Additionen organometallischer Reagentien an Cyclopentanone niedriger ausfallen als bei Additionen an ähnliche Cyclohexanone. Wegen der größeren Enolisierungstendenz von Cyclopentanonen sind auch die chemischen Ausbeuten im allgemeinen niedriger. Die entsprechenden Organocerreagentien wurden in situ durch einstündiges Rühren der Cerverbindungen [siehe Gl. (a)-(c)] mit Lithiumorganylen bei $-78^{\circ} \mathrm{C}$ gebildet. Um die Additionsprodukte $\mathrm{rac}-\mathbf{2}$ und $\mathrm{rac}-\mathbf{3} \mathrm{zu}$ erhalten, wurde
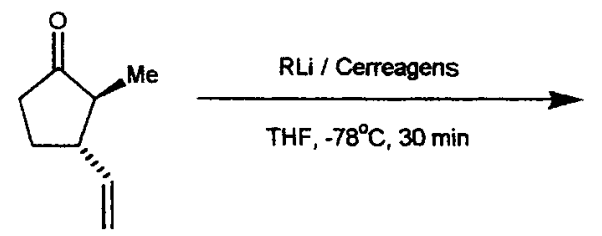

rac-1

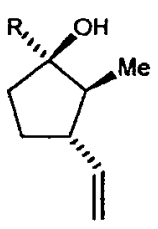

rac-2 Hauptdiastereomer

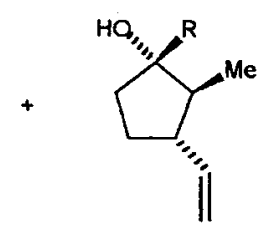

rac-3 Nebendiastereomer anschließend 2-Methyl-3-vinylcyclopentanon rac-1 ${ }^{[8]}$ bei dieser Temperatur zugegeben. Die Ergebnisse sind in Tabelle 1 zusammengefaßt. Die Struktur der Organocerspezies ist bislang unbekannt und konnte noch nicht aufgeklärt werden. ${ }^{[9]}$ Wahrschein-

Tabelle 1. Stereoselektive Additionen von Cerorganylen an 2-Methyl-3-vinylcyclopentanon rac-1 [12].

\begin{tabular}{lllll}
\hline Nr. & Reagens & $d e[\%][\mathrm{a}]$ & Ausb. [\%] & Enolisierung[\%] \\
\hline 1 & $\mathrm{MeLi}$ & 10 & 65 & 22 \\
2 & $\mathrm{MeLi} / \mathrm{CeCl}$ & 68 & 91 & - \\
3 & $\mathrm{MeLi} / \mathrm{Ce}\left(\mathrm{NEt}_{2}\right)_{3}$ & 78 & 55 & 25 \\
4 & $\mathrm{MeLi} / \mathrm{Ce}\left(\mathrm{NiPr}_{2}\right)_{3}$ & 79 & 50 & 36 \\
5 & $\mathrm{MeLi} / \mathrm{Ce}(\mathrm{OiPr})_{3}$ & 83 & 95 & - \\
6 & $\mathrm{MeLi} / \mathrm{Ce}(\mathrm{O} i \mathrm{Pr})_{3}[\mathrm{~b}]$ & 86 & 93 & - \\
7 & $\mathrm{MeLi} / \mathrm{Ce}(\mathrm{OiPr})_{3}[\mathrm{c}]$ & 74 & 90 & - \\
8 & $\mathrm{MeLi} / \mathrm{ClCe}(\mathrm{O} t \mathrm{Bu})_{2}$ & 77 & 85 & - \\
9 & $\mathrm{MeLi} / \mathrm{ClCe}\left(\mathrm{OCH} i \mathrm{Pr}_{2}\right)_{2}$ & 87 & 89 & - \\
10 & $n \mathrm{BuLi}$ & 49 & 56 & 36 \\
11 & $n \mathrm{BuLi} / \mathrm{ClCe}\left(\mathrm{OCH} i \mathrm{Pr}_{2}\right)_{2}$ & 89 & 90 & - \\
12 & $t \mathrm{BuLi}$ & 77 & 35 & 57 \\
13 & $t \mathrm{BuLi} / \mathrm{ClCe}\left(\mathrm{OCH} i \mathrm{Pr}_{2}\right)_{2}$ & 90 & 80 & - \\
14 & $\mathrm{PhLi}$ & 85 & 70 & 24 \\
15 & $\mathrm{PhLi} / \mathrm{ClCe}\left(\mathrm{OCH} i \mathrm{Pr}_{2}\right)_{2}$ & 94 & 95 & - \\
& & &
\end{tabular}

[a] Durch GC-Analyse bestimmt. [b] Addition bei $-98^{\circ} \mathrm{C}$. [c] In $\mathrm{Et}_{2} \mathrm{O}$.

lich handelt es sich bei den aus den Cerchloriddialkoxiden erhaltenen Organocerverbindungen um eine ungeladene Spezies des Typs $\mathrm{RCe}\left(\mathrm{OR}^{\prime}\right)_{2}$. Ausgehend von den Cer(III)trialkoxiden sollten dagegen Komplexe der allgemeinen Formel $\mathrm{Li}\left[\mathrm{CeR}\left(\mathrm{OR}^{\prime}\right)_{3}\right]$ resultieren.

Eine Enolisierung der Ketone wurde ausschließlich bei der Reaktion mit Organocerverbindungen festgestellt, die sich von den Certriamiden ableiten (Tabelle 1, Nr. 3 und 4). Die Liganden üben einen deutlichen Einfluß auf die Stereoselektivität der Addition aus. Wie erwartet, führte zunehmende sterische Hinderung auch zu erhöhter Diastereoselektivität. Im Falle der sterisch besonders anspruchsvollen Organocerverbindungen $\mathrm{Li}$ $\left[\mathrm{CeMe}(\mathrm{O} t \mathrm{Bu})_{3}\right]$ und $\mathrm{Li}\left[\mathrm{CeMe}\left(\mathrm{OCH} i \mathrm{Pr}_{2}\right)_{3}\right]$ fand jedoch keine Reaktion mehr mit dem Substrat statt. Die Diastereoselektivität ließ sich durch Erniedrigung der Reaktionstemperatur auf $-98^{\circ} \mathrm{C}$ nur geringfügig steigern (Nr. 6). Sie war bei Verwendung von Diethylether als Solvens niedriger als bei Verwendung von THF (Nr. 7). Die besten Resultate konnten in THF mit $\mathrm{RCe}\left(\mathrm{OCH} i \mathrm{Pr}_{2}\right)_{2}$ erreicht werden. Dessen Addition an rac-1 (Nr. 9, 11, 13 und 15) verlief mit für die Addition metallorganischer Reagentien an Cyclopentanone ${ }^{[10,11]}$ guten bis sehr guten Diastereoselektivitäten $(87-94 \%$ de).

Um die Anwendungsbreite der neuen Cerverbindungen in Carbonyladditionen zu dokumentieren, wurde die Addition der von $\mathrm{ClCe}\left(\mathrm{OCH} i \mathrm{Pr}_{2}\right)_{2}$ und $\mathrm{Ce}\left(\mathrm{OCH} \mathrm{Pr}_{2}\right)_{3}$ abgeleiteten Organocerreagentien an 2-Methylcyclohexanon, 4-tert-Butylcyclohexanon und 2-Phenylpropionaldehyd (Crams Aldehyd) untersucht (Tabelle 2). Bei der Reaktion von Li[CeMe $\left.\left(\mathrm{OCH} i \mathrm{Pr}_{2}\right)_{3}\right]$ mit 2-Methylcyclohexanon fand, wie auch mit 2-Methyl-3-vinylcyclopentanon $\mathrm{rac}$-1, keine Addition statt. Hingegen lieferte die Addition von $\mathrm{MeCe}\left(\mathrm{OCH}_{i} \mathrm{Pr}_{2}\right)_{2}$ und $n \mathrm{BuCe}\left(\mathrm{OCH} i \mathrm{Pr}_{2}\right)_{2}$ an 2-Methylcyclohexanon die entsprechenden Alkohole mit Diastereoselektivitäten von 97 bzw. $98 \%$ de, bei Verwendung der sterisch weniger anspruchsvollen Organocerverbindungen $\mathrm{MeCeCl}_{2}$ und $n \mathrm{BuCeCl}_{2}$ sanken die Selektivitäten auf 88 bzw. $94 \%$ de. Die Addition des sterisch sehr anspruchsvollen Li[CeMe$\left(\mathrm{OCH} i \mathrm{Pr}_{2}\right)_{3}$ ] sowohl an 4-tert-Butylcyclohexanon als auch an 2-Phenylpropionaldehyd gelang mit Ausbeuten von 88 bis $89 \%$. Bei 4-tert-Butylcyclohexanon wurde eine Erhöhung der Diaste- 
Tabelle 2. Stereoselektive Addition von Cerorganylen an ausgewähite Carbonylsubstrate.

\begin{tabular}{llll}
\hline Carbonylverbindung & Reagens & $d e[\%][\mathrm{a}]$ & Ausb. [\%] \\
\hline 2-Methylcyclohexanon & $\mathrm{MeLi}$ & 63 & 74 \\
2-Methylcyclohexanon & $\mathrm{MeCeCl}$ & 88 & 89 \\
2-Methylcyclohexanon & $\mathrm{MeCe}\left(\mathrm{OCH}_{2} \mathrm{Pr}_{2}\right)_{2}$ & 97 & 91 \\
2-Methylcyclohexanon & $\mathrm{Li}\left[\mathrm{CeMe}\left(\mathrm{OCH} i \mathrm{Pr}_{2}\right)_{3}\right]$ & - & $-[\mathrm{b}]$ \\
2-Methylcyclohexanon & $n \mathrm{BuLi}$ & 78 & 80 \\
2-Methylcyclohexanon & $n \mathrm{BuCeCl}$ & 94 & 92 \\
2-Methylcyclohexanon & $n \mathrm{BuCe}\left(\mathrm{OCH}_{2} \mathrm{Pr}_{2}\right)_{2}$ & 98 & 88 \\
4-tert-Butylcyclohexanon & $\mathrm{MeLi}$ & 24 & 50 \\
4-tert-Butylcyclohexanon & $\mathrm{MeCeCl}$ & 36 & 93 \\
4-tert-Butylcyclohexanon & $\left.\mathrm{LiCeMe}\left(\mathrm{OCH} i \mathrm{Pr}_{2}\right)_{3}\right]$ & 68 & 88 \\
2-Phenylpropionaldehyd & $\mathrm{MeLi}$ & 82 & 57 \\
2-Phenylpropionaldehyd & $\mathrm{MeCeCl}$ & 84 & 85 \\
2-Phenylpropionaldehyd & $\mathrm{Li}\left[\mathrm{CeMe}\left(\mathrm{OCH} i \mathrm{Pr}_{2}\right)_{3}\right]$ & 90 & 89 \\
\hline
\end{tabular}

[a] Durch GC-Analyse bestimmt. [b] Addition fand nicht statt.

reoselektivität von $36\left(\mathrm{MeCeCl}_{2}\right)$ auf $68 \%$ de (Li[CeMe$\left.\left.\left(\mathrm{OCH} \mathrm{Pr}_{2}\right)_{3}\right]\right)$ festgestellt. Dessen Addition an 2-Phenylpropionaldehyd lieferte mit einer Diastereoselektivität von $90 \%$ de den entsprechenden Alkohol. Demgegenüber führte die Verwendung von $\mathrm{MeCeCl}_{2}(84 \%$ de) oder $\mathrm{MeLi}(82 \%$ de) zu deutlich geringeren Selektivitäten. Die Anwendung der neuen Organocerreagentien in der Naturstoffsynthese, z. B. für die enantioselektive Synthese des antifungal wirksamen Chokols, ${ }^{[10,13]}$ wird derzeit von uns untersucht.

\section{Experimentelles}

Alle Reaktionen wurden mit Schlenktechnik oder in einem Handschuhkasten unter striktem Sauerstoff- und Wasserausschluß durchgeführt.

Herstellung der Certrialkoxide: Nach Zugabe von $20 \mathrm{~mL}$ des jeweiligen Alkohols zu einer Lösung von $6.0 \mathrm{mmol} \mathrm{Ce}(\mathrm{OiPr})_{3}$ in $10 \mathrm{~mL}$ Benzol bei Raumtemperatur wurde der Ansatz $15 \mathrm{~h}$ unter Rückfluß erhitzt. Anschließend wurde das Reaktionsgemisch auf Raumtemperatur abgekühlt und das Lösungsmittel im Vakuum entfernt $\left(20^{\circ} \mathrm{C}\right.$, 0.005 Torr). Die Certrialkoxide wurden in Ausbeuten von 90-95\% isoliert.

Herstellung der Cerchloriddialkoxide: Zu einer Lösung der entsprechenden Certrialkoxide in $20 \mathrm{~mL}$ Benzol wurden bei Raumtemperatur $2.0 \mathrm{mmol}$ Acetylchlorid tropfenweise zugegeben. Nach beendeter Zugabe fiel ein brauner Niederschlag aus Zur Vervollständigung der Reaktion wurde $2 \mathrm{~h}$ unter Rückfluß erhitzt und nach Abkühlen auf Raumtemperatur die überstehende Lösung über eine Kanüle entfernt. Der Rückstand wurde im Vakuum getrocknet $\left(20^{\circ} \mathrm{C}, 0.005\right.$ Torr $)$, und die Cerchloriddialkoxide wurden in Ausbeuten von $80-90 \%$ isoliert

Carbonyladditionen: $\mathrm{Zu}$ einer Lösung von $1.5 \mathrm{mmol}$ der Cerverbindung in $5 \mathrm{~mL}$ Tetrahydrofuran wurden bei $-78^{\circ} \mathrm{C} 1.2 \mathrm{mmol}$ Methyllithium $(0.75 \mathrm{~mL}$ einer $1.6 \mathrm{M}$ Lösung in Diethylether) tropfenweise zugegeben. Nach $1 \mathrm{~h}$ wurde $1.0 \mathrm{mmol}$ der entsprechenden Carbonylkomponente langsam hinzugegeben und das Reaktionsgemisch weitere $30 \mathrm{~min}$ bei dieser Temperatur gerührt. Nach Zugabe von $10 \mathrm{~mL}$ einer gesättigten Ammoniumchloridlösung wurde zur Entfernung ausgefallener Salze über eine kurze Schicht Celite filtriert und mit Diethylether nachgewaschen. Die vereinigten organischen Phasen wurden über Magnesiumsulfat getrocknet, anschließend wurde das Solvens im Vakuum entfernt und der Rückstand an Kieselgel chromatographisch gereinigt.

Stichwörter: Asymmetrisch-Synthese $\cdot$ Cer - Cycloalkanone • Lanthanoide - Ligandeneffekte

[1] a) G. A. Molander, Chem. Rev. 1992, 92, 29-68; b) T. Imamoto in Comprehensive Organic Synthesis, Vol. 1 (Hrsg.: B. M. Trost, I. Fleming, S. L. Schreiber), Pergamon, Oxford, 1991, S. 231-250.

[2] F. A. Cotton, G. Wilkinson, Anorganische Chemie, 4. Aufl., VCH, Weinheim, 1982.

[3] a) M. T. Reetz, Organotitanium Reagents in Organic Synthesis, Springer, Berlin, 1986; b) M. T. Reetz, N. Harmat, R. Mahrwald, Angew. Chem. 1992, 104 333-334; Angew. Chem. Int. Ed. Engl. 1992, 31,342-344; c) M. T. Reetz, H. Haning, S. Stanchev, Tetrahedron Lett. 1992, 33, 6963-6966; d) K. Chibale, N. Greeves, L. Lyford, J. E. Pease, Tetrahedron: Asymmetry 1993, 4, 2407-2410 e) N. Greeves, L. Lyford, J. E. Pease, Tetrahedron Lett. 1994, 35, 385-388; f) N. Greeves, J. E. Pease, M. C. Bowden, S. M. Brown, ibid. 1996, 37, 26752678; g) N. Greeves, J. E. Pease, ibid. 1996, 37, 5821-5824.
[4] a) A. Lebrun, J. L. Namy, H. B. Kagan, Tetrahedron Lett. 1991, 32, 23552358 ; b) verbesserte Synthese von $\mathrm{Ce}(\mathrm{OiPr})_{3}:$ P. Eckenberg, U. Groth, T. Köhler, Liebigs Ann. Chem. 1994, 673-677.

[5] In Analogie zur Synthese von $\mathrm{Gd}(\mathrm{OR})_{3}$ : R. C. Mehrotra, J. M. Batwara, Inorg. Chem. 1970, 9, 2505-2510.

[6] In Analogie zur Synthese von CISm(OR) 2 : B. S. Sankhla, R. N. Kapoor, Can. J. Chem. 1966, 44, 2131-2137.

[7] In Analogie zur Synthese von Ce[N(SiMe $\left.)_{2}\right]_{3}:$ D. C. Bradley, J. S. Ghorta, F. A. Hart, J. Chem. Soc. Dalton Trans. 1973, 1021-1023.

[8] G. Quinkert, W.-D. Weber, U. Schwartz, H. Stark, H. Baier, G. Frank, G. Dürner, Liebigs Ann. Chem. 1981, 2335-2371.

[9] a) S. E. Denmark, J. P. Edwards, O. Nicaise, J. Org. Chem. 1993, 58, 569-578; b) W. J. Evans, J. D. Feldman, J. W. Ziller, J. Am. Chem. Soc. 1996, 118, 45814584.

[10] a) E. A. Mash, J. Org. Chem. 1987, 52, 4143-4146; b) U. Groth, W. Halfbrodt, T. Köhler, P. Kreye, Liebigs Ann. Chem. 1994, 885-890.

[11] G. A. Molander, E. R. Burkhardt, P. Weinig, J. Org. Chem. 1990, 55, 4990 4991.

[12] 2-Methyl-3-vinylcyclopentanon wurde als untrennbares Gemisch der cis/transIsomere im Verhältnis 7:93 erhalten. Aus diesem Grunde resultieren 5-7\% der Alkohole aus einem $\beta$-Angriff der Organocerverbindung an dic CarbonylGruppe des Ketons cis-1, siehe Lit. [10].

[13] E. Urban, G. Knühl, G. Helmchen, Tetrahedron 1995, 52, $13031-13038$. 